\title{
AMELIORATIVE EFFECTS OF DIMETHYL FUMARATE IN ACUTE AND CHRONIC MURINE MODELS OF DEPRESSION
}

\author{
LORETTA O. INIAGHE ${ }^{1 *}$, ELOHO E. OKPAKPOR ${ }^{1}$, OSAZE O. EDOSUYI ${ }^{1}$, \\ EWERE O. ESEKA ${ }^{1}$ AND OGUGUA I. NEDOZI ${ }^{1}$
}

\author{
${ }^{1}$ Department of Pharmacology and Toxicology, Faculty of Pharmacy, University of Benin, Nigeria
}

\begin{abstract}
Depression, a major contributor to the global disease burden, occurs in both adults and children and is associated with impaired antioxidant defense mechanisms. This study examined the effects of dimethyl fumarate (DMF), a fumaric acid ester with immunomodulatory, anti-inflammatory and antioxidant properties in murine models of depression. In the first phase of the study, mice were treated with either the vehicle, 50 and $100 \mathrm{mg} / \mathrm{kg}$ DMF or $15 \mathrm{mg} / \mathrm{kg}$ imipramine and subjected to either the forced swim or tail suspension tests. Thereafter, mice were euthanized and levels of antioxidant markers in isolated brain tissues were assayed. In the second phase of the study, mice were subjected to the chronic unpredictable mild stress regimen for four weeks and treated with the vehicle, DMF or imipramine in the last two weeks of the stress protocol. Forced swim and percentage sucrose preference tests were used for behavioral evaluations. Mice were sacrificed, brain levels of catalase, glutathione, thiobarbituric reactive acid substance and nitrite were quantified. Treatment with DMF significantly $(\mathrm{p}<0.05)$ reduced periods of immobility in both the forced swim and tail suspension tests following acute and chronic drug treatment and improved sucrose consumption after exposure to chronic unpredictable mild stress. DMF also significantly improved $(\mathrm{p}<0.05)$ levels of antioxidants (catalase and glutathione) while reducing prooxidant biomarkers (thiobarbituric reactive acid substance and nitrite levels) in the brain. DMF ameliorated depression in mice and augmented the antioxidant defense mechanism in the brains of mice subjected to chronic unpredictable mild stress.
\end{abstract}

Keywords: forced swim test, chronic unpredictable mild stress, catalase, sucrose preference, antioxidant, thiobarbituric reactive acid substance

Depression, a major contributor to the universal global burden of disease occurs both in children as young as three years of age and in adults. Depression which was ranked to be the fourth leading cause of disease in 2007, is projected-together with ischaemic heart diseases and road traffic accidents- to be one of the three major contributors to the global disease by the year $2020(1,2)$. Depression is also an important global public health issue, because of the relatively high lifetime prevalence, association with substantial disability and its comorbidity with chronic physical diseases such as stroke, diabetes, arthritis and multiple sclerosis $(2,3)$.

The increase in the number of antidepressants, unfortunately, does not correlate with the pharmacotherapy of depression and patients are often refractory to treatment. The side effects associated with antidepressant therapy which limit patients' adherence to therapy vary in intensity from mild to moderate and include apathy, fatigue, sleep distur- bances and cognitive impairment $(4,5)$. The need for development of pharmacological agents with minimal side effects cannot be overemphasized.

Biochemical studies have shown that brain oxidative damage is involved in the pathogenesis of depression. Antioxidant profiles of depressed patients were demonstrated to be different from those of healthy patients with significantly higher lipid peroxidation products and lower levels of superoxide dismutase, glutathione, and catalase. Antidepressant agents were also reported to reverse the increase in oxidative load associated with depressive episodes $(4,6,7)$.

Animal models continue to be important tools for investigating possible causes and treatment for human diseases, and several models of depression have been proposed to evaluate potential antidepressant effects of test compounds following acute and chronic drug therapy $(8,9)$. The forced swim test (FST) is the most widely used acute animal model for antidepressant screening in many laboratories

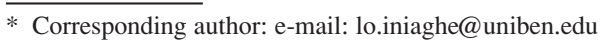


due to its advantages of being affordable, simple, reliable and sensitive to a broad spectrum of clinically effective antidepressants (10). The tail suspension test (TST), similar to the FST also detects a broad spectrum of antidepressants and is often used with the FST to obtain complementing or converging data $(11,12)$. The chronic unpredictable mild stress (CUMS) model is a valid and widely used chronic model of depression based on loss of responsiveness to rewards by animals subjected to varying schedule of minor stressors $(13,14)$. The CUMS simulates both behavioral and biochemical features of human depression such as anhedonia, behavioral helplessness and increased production of reactive oxygen species $(5,7,15,16)$

Dimethyl fumarate (DMF) is a methyl ester of fumaric acid which has been shown to possess immunomodulatory, anti-inflammatory, antioxidant and neuroprotective effects in the central nervous system in both pre-clinical and clinical studies and is currently used in the treatment of remitting, relapsing multiple sclerosis (17-19). In in-vitro studies of oxidative stress, DMF prevented oxidative damage by up-regulating haem oxygenase-1 (HO-1) enzyme (20); while in a murine models of intracerebral hemorrhage, treatment with DMF unregulated the expression of phosphorylated nuclear factor erythroid- 2 derived factor-2, HO-1, catalase and nicotinamide adenine dinucleotide phosphate quinone oxidoreductase genes $(19,21)$.

Considering the role of DMF in oxidative stress, this study was therefore carried out to investigate (i) antidepressant potential of DMF in acute and chronic murine behavioral models of depression (ii) antioxidant profile of mice subjected to FST, TST, and CUMS and treated with DMF.

\section{EXPERIMENTAL}

\section{Reagents/Chemicals}

Dimethylsulphoxide (DMSO), dimethyl fumarate, bovine serum albumin, thiobarbituric acid and 5,5'-dithiobis-(2-nitrobenzoic acid)were purchased from Sigma Aldrich, St. Louis, USA. Sodium hydroxide, hydrochloric acid, tris hydrochloride, sulphosalicylic acid, trichloroacetic acid, imipramine, 99\% chloroform, Greiss Reagent, sodium chloride, disodium hydrogen phosphate and dihydrogen phosphate were purchased from JHD Chemicals, Guangdong, China.

\section{Animals}

Swiss albino male mice weighing 18-26 g procured from the University of Benin Animal House,
Benin City, Edo State, were used for the study. They were kept in plastic cages at the Animal House of the Department of Pharmacology and Toxicology, University of Benin, Benin City. They were maintained under standard controlled environment and were allowed free access to feed (Top feeds ${ }^{\circledast}$ Growers Mash, Super-Deluxe Animal Feed Mills Co. Ltd, Nigeria) and clean water ad libitum. Handling of the animals was done according to standard protocols for the use of laboratory animals of the National Institute of Health (22) and ethical approval from the Institutional Ethics Committee was obtained for the study (EC/FP/018/09). Efforts were made to minimize suffering. Behavioral tests were carried out between 9.00 to 17.00 hours daily and separate groups of animals were used for the neurobehavioral tests.

\section{Evaluation of antidepressant activity of DMF}

The study was carried out using acute and chronic models of depression.

\section{Acute study: forced swim test}

The method of Porsolt et al. (23) with some modifications was used. Twenty-four mice were randomly distributed into four groups of six mice per group. The first group which served as control were given the vehicle (DMSO and distilled water) orally; the second and third group received 50 $\mathrm{mg} / \mathrm{kg}$ and $100 \mathrm{mg} / \mathrm{kg}$ of DMF orally respectively, while the fourth group received the aqueous solution of imipramine $(15 \mathrm{mg} / \mathrm{kg})$ intraperitoneally.

One hour post administration, each mouse was placed in a transparent cylindrical container with a diameter of $26.1 \mathrm{~cm}$ and height of $24.8 \mathrm{~cm}$ containing water at a depth of $15 \mathrm{~cm}$ and a temperature of $25^{\circ} \mathrm{C}$ for six minutes. The period of immobility in the last four minutes of the experiment was cumulatively recorded by trained observers blinded to treatment. After each test, the mice were properly dried before they were returned to their home cages. A mouse was judged to be immobile when it made movements only necessary to keep its head above water; the mice were subjected to a 15-minute training session under similar conditions, 24 hours before the test.

Twenty four hours after drug administration and behavioral tests, animals were sacrificed under chloroform anesthesia and whole brains carefully isolated.

\section{Tail suspension test}

For the tail suspension test, twenty-four mice were randomly divided into four groups. The control 
and standard drug treatment groups received the vehicle (p.o.) and imipramine (15 mg/kg i.p.) respectively; while test groups were treated with 50 and $100 \mathrm{mg} / \mathrm{kg}$ DMF p.o (11). One hour following drug administration, each mouse was suspended from the edge of a shelf $60 \mathrm{~cm}$ above a table top for six minutes. The mice were suspended by adhesive tape placed approximately $1 \mathrm{~cm}$ from the tip of the tail. Periods of immobility were recorded by trained observers blinded to treatment.

Twenty-four hours after the TST, animals were euthanized under chloroform anesthesia and whole brains were carefully isolated.

\section{Biochemical evaluation}

Twenty-four hours after either after the FST or TST, animals were anesthetized, euthanized and brains carefully isolated. The brains were washed with phosphate buffer ( $\mathrm{pH} 7.4)$, homogenized, centrifuged and supernatant layers collected and used for biochemical assays. A separate group of mice not subjected to either the FST, TST or drug administration were also sacrificed and the brains were isolated for biochemical analysis.

\section{Chronic study: chronic unpredictable mild stress}

The Chronic unpredictable mild stress (CUMS) protocol as described by Katz (13) and Willner et al. (14) with some modifications was used. Before the start of the study, animals were placed on the rotarod (Ugobasile, Comerio Italy; 47600 V04) set at a constant speed of $20 \mathrm{rpm}$ to eliminate possible false negatives arising from unhealthy or possibly neurologically impaired animals. Animals which fell off the horizontal bar in less than 30 seconds were excluded from the study. Animals were exposed daily to various unpredictable stressors none of which was sufficient to produce long-term effects (5).

Fifty-six mice randomly distributed into six groups of nine animals each were subjected to the following regimen:

Group I: Non-CUMS group

Group II: CUMS only

Group III: CUMS + Vehicle

Group IV: CUMS + DMF $50 \mathrm{mg} / \mathrm{kg}$

Group V: CUMS + DMF $100 \mathrm{mg} / \mathrm{kg}$

Group VI: CUMS + Imipramine $15 \mathrm{mg} / \mathrm{kg}$.

The CUMS protocol was carried out for 4 successive weeks and drug administration was done in the last 2 weeks of the regimen. The CUMS procedure consisted of exposure to wet beddings for 4 hours (2 times), exposure to wet beddings for 6 hours (once), exposure to foreign objects (once), cage tilting at $45^{\circ}$ for 4 hours ( 2 times), cage tilting at $45^{\circ}$ for 6 hours ( 3 times), food deprivation for 6 hours (once), food deprivation for 24 hours (3 times), water deprivation for 8 hours (once), water deprivation for 24 hours (2 times), exposure to Wister rats in a restrainer for 2 hours (once), isolation for 24 hours ( 3 times), exposure to cages with rat odour (once), exposure to cages with female odour and beddings from female cages (once), restraint for 2 hours (2times), 10 minutes shaker stress (3 times), 5 minutes exposure to heat stress at $50^{\circ}$ (once). The sequence and duration of stressors mice were exposed to were varied daily in order to reduce the levels of predictability. The CUMS protocol adopted is illustrated in Table 1.

Table 1. Protocol of daily stressors and duration of stressors in the CUMS

\begin{tabular}{|c|c|c|}
\hline Day & Stressor & Duration \\
\hline 1 & Wet beddings & $4 \mathrm{~h}$ \\
\hline 2 & Foreign objects & $2 \mathrm{~h}$ \\
\hline 3 & Cage tilting at $45^{\circ}$ & $4 \mathrm{~h}$ \\
\hline 4 & Food deprivation & $6 \mathrm{~h}$ \\
\hline 5 & Water deprivation & $8 \mathrm{~h}$ \\
\hline 6 & Wister rat exposure & $2 \mathrm{~h}$ \\
\hline 7 & Isolation & $24 \mathrm{~h}$ \\
\hline 8 & Food deprivation & $24 \mathrm{~h}$ \\
\hline 9 & Restraint & $2 \mathrm{~h}$ \\
\hline 10 & Shaker stress & $10 \mathrm{~min}$ \\
\hline 11 & Cages with rat odor & $8 \mathrm{~h}$ \\
\hline 12 & Cage tilting at $45^{\circ}$ & $4 \mathrm{~h}$ \\
\hline 13 & Wet beddings & $4 \mathrm{~h}$ \\
\hline 14 & Water deprivation & $24 \mathrm{~h}$ \\
\hline 15 & Shaker stress & $10 \mathrm{~min}$ \\
\hline 16 & Cages with female beddings & $8 \mathrm{~h}$ \\
\hline 17 & Cage tilting at $45^{\circ}$ & $6 \mathrm{~h}$ \\
\hline 18 & Isolation & $24 \mathrm{~h}$ \\
\hline 19 & Food deprivation & $24 \mathrm{~h}$ \\
\hline 20 & Heat Stress & $10 \mathrm{~min}$ \\
\hline 21 & Cage tilting at $45^{\circ}$ & $6 \mathrm{~h}$ \\
\hline 22 & Water deprivation & $24 \mathrm{~h}$ \\
\hline 23 & Restraint & $2 \mathrm{~h}$ \\
\hline 24 & Shaker stress & $10 \mathrm{~min}$ \\
\hline 25 & Food deprivation & $24 \mathrm{~h}$ \\
\hline 26 & Isolation & $24 \mathrm{~h}$ \\
\hline 27 & Wet beddings & $6 \mathrm{~h}$ \\
\hline 28 & Cage tilting at $45^{\circ}$ & $6 \mathrm{~h}$ \\
\hline
\end{tabular}


Thereafter, mice were subjected to neurobehavioral evaluations and sacrificed for isolation of tissues.

\section{Forced swim test}

The forced swim test was carried out as previously described. Animals were placed in a transparent cylindrical container and allowed to swim for six minutes. The period of immobility in the last four minutes of the experiment was cumulatively recorded by observers blinded to treatment. The mice were subjected to a 15-minute training session under similar conditions, 24 hours before the test (23).

\section{Sucrose preference test}

The sucrose preference test (SPT) which is a measure of anhedonia and is based on rodents' intrinsic love for sweet solutions was conducted in 3 phases as described by Willner et al. (24) and Serchov et al. (25). In phase 1 , the habituation phase, tap water in the home cages was replaced with $1 \% \mathrm{w} / \mathrm{v}$ sucrose solution for 24 hours. This was followed by phase 2 in which each mouse was transferred to individual cages and given both tap water and sucrose solution for 3 days to obtain sucrose preference baseline. In the final phase, the sucrose preference test, mice were given both $1 \% \mathrm{w} / \mathrm{v}$ solution and tap water for 24 hours. Volume of fluid consumed (tap bottle or sucrose) was determined by calculating the difference between the weight of the bottle before and after exposure. The sucrose preference was calculated using the formula

$\%$ Preference $=($ sucrose intake $/$ total intake $) \cdot 100$

\section{Biochemical evaluation}

Twenty four hours after the neurobehavioral tests, animals were anesthetized with chloroform and sacrificed, whole brains were isolated, washed carefully, homogenized and centrifuged in phosphate buffer ( $\mathrm{pH} \mathrm{7.4)}$ for $30 \mathrm{~min}$ at $12000 \mathrm{rpm}$. Supernatant fractions were separated and used for biochemical assays while the pellet fractions were discarded.

\section{Protein determination assay}

Protein was measured in brain homogenate using bovine serum albumin as standard according to the method described by Lowry et al (26).

\section{Estimation of brain catalase levels}

Catalase levels in the brain was assayed following the method of Claiborne (27). Hydrogen peroxide $(1 \mathrm{~mL})$ was added to the assay mixture made up of phosphate buffer (1.95 mL, pH 7.4) and brain homogenate $(0.05 \mathrm{~mL})$ and changes in absorbance were immediately recorded at $240 \mathrm{~nm}$ for each sample. Catalase levels obtained are expressed as micromoles of hydrogen peroxide consumed per minute per milligram of protein.

\section{Estimation of brain glutathione levels}

Following the method of Ellman (28), $1 \mathrm{~mL}$ of brain homogenate was added to $1 \mathrm{~mL}$ of $4 \%$ sulphosalicylic acid and kept at $4^{\circ} \mathrm{C}$ for 1 hour. The mixture was centrifuged for 15 minutes at $2000 \mathrm{rpm}$, and supernatant layer separated. Phosphate buffer (2.7 mL, pH 7.4) and 0.2 mL 5,5'-dithiobis (2nitrobenzoic acid) were added to $1 \mathrm{~mL}$ of the supernatant and the resultant colour changes were measured at $412 \mathrm{~nm}$.

\section{Estimation of brain lipid peroxidation}

Malondialdehyde content which a measure of lipid peroxidation was estimated in the form of thiobarbituric reactive acid substance according to the method described by Wills (29). A mixture of brain homogenate $(0.5 \mathrm{~mL})$ and tris hydrochloric acid $(0.5$ $\mathrm{mL}$ ) was incubated at room temperature for 2 hours. Trichloroacetic acid $(1 \mathrm{~mL}, 10 \%)$ was then added and the mixture was centrifuged at $1500 \mathrm{~g}$ for 10 minutes; the supernatant layers carefully separated. To $1 \mathrm{~mL}$ of the resultant supernatant, an equal volume of thiobarbituric acid $(1 \mathrm{~mL})$ was added and the mixture was boiled for 10 minutes and allowed to cool. Distilled water $(1 \mathrm{~mL})$ was then added and the absorbance changes were measured at a wavelength of $532 \mathrm{~nm}$ using an ultraviolet/visible spectrophotometer. A calibration curve using malondialdehyde was generated and the results obtained are expressed as nanomoles of MDA per milligram of protein.

\section{Estimation of Brain nitrite levels}

Greiss reagent, an indicator of nitric oxide production was used to determine nitrite levels according to the protocol described by Green et al. (30). An equal volume of Greiss reagent $(500 \mu \mathrm{L})$ was added to the $500 \mu \mathrm{L}$ of brain homogenate and the mixture was incubated in the dark for 10 minutes at room temperature. Absorbance was measured at $546 \mathrm{~nm}$ and nitrite levels obtained are expressed as micromole per milligram of protein.

\section{Statistical analysis}

The results were analyzed for statistical significance using one-way analysis of variance (ANOVA) followed by Student-Newman-Keuls test using Sigma ${ }^{\circledast}$ Stat version version 11.0. A difference was considered significant at $p<0.05$. The results 
are presented as a mean \pm standard error of the mean (SEM).

\section{RESULTS}

\section{Evaluations after acute drug administration Behavioral assays: forced swim and tail suspen- sion tests}

DMF significantly reduced periods of immobility in the forced swim and tail suspension tests following acute administration compared to the vehicle-treated animals. Periods of immobility in DMF treated animals were however not significantly different from imipramine treated animals. Data is presented in Figure 1A and B.

\section{Biochemical assays}

Following acute treatment, there was no significant difference in levels of catalase, GSH, TBARS and nitrite levels across all the intervention groups. Data is presented in Figure 2A-D.

\section{Evaluations after chronicdrug administration Behavioral assays}

Data from the FST following CUMS and chronic drug treatment are presented in Figure 3A. There was an increase in periods of immobility in the CUMS and vehicle-treated animals which was significantly different from DMF, non-CUMS and imipramine treated groups. There was, however, no significant difference between DMF treated, nonCUMS and imipramine treated animals.

\section{Sucrose preference test}

An increase in preference for sucrose solution was observed high dose DMF $(100 \mathrm{mg} / \mathrm{kg})$ treated animals, Imipramine and non-CUMS groups compared to the CUMS and vehicle-treated animals.
Low dose DMF had an increase in sucrose consumption but this failed to achieve significance when compared to non-CUMS, Imipramine and high dose DMF treatment groups (Fig. 3B).

\section{Biochemical assays}

Catalase levels

Levels of catalase enzymes in brains of mice subjected to CUMS were significantly lower $(\mathrm{p}<$ 0.05 ) than those subjected to CUMS and treated with 2 dose levels of DMF and imipramine. Vehicle-treated animals showed no appreciable increase in catalase levels. These results are presented in Figure 4A.

\section{Glutathione levels}

Animals subjected to CUMS protocol had significantly lower levels of GSH compared to the nonCUMS groups. Chronic treatment with imipramine and DMF reversed this decrease (Fig. 4B).

\section{Lipid peroxidation}

Levels of MDA- a lipid peroxidation product measured in form of TBARS- were different in the various intervention groups. Treatment with imipramine reduced the activity of a thiobarbituric reactive acid substance in brain homogenates of animals subjected to imipramine following the CUMS protocol. This was not significantly different from both doses of DMF and non-CUMS treated groups but significantly different from vehicle and CUMS animals. Data is shown in Figure 4C.

\section{Nitrite levels}

Nitrite levels in DMF and Imipramine groups were lower than those of vehicle and non-CUMS groups, no significant difference was observed between the various intervention groups and nonCUMS animals (Fig. 4D).

\section{$\mathrm{A}$}

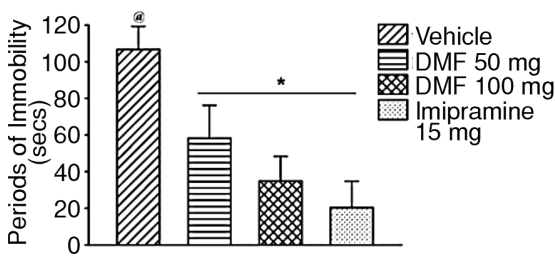

B

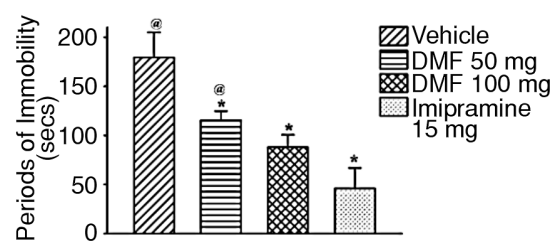

Figure 1. Statistical analysis of periods of immobility in the forced swim test (A) and tail suspension test (B) following treatment with DMF. DMF reduced periods of immobility in both tests. Data is expressed as mean \pm SEM. ${ }^{*} \mathrm{p}<0.05$ compared to the vehicle, ${ }^{@} \mathrm{p}<0.05$ compared to imipramine; $n=6$ per group 


\section{DISCUSSION AND CONCLUSION}

In this study, treatment with DMF: (i) reduced periods of immobility in the FST and TST but did not affect nitrite levels, catalase, GSH and TBARS following acute drug treatment (ii) reduced periods of immobility in the FST postexposure to CUMS and chronic drug treatment (iii) increased levels of sucrose consumption postexposure to CUMS and chronic drug treatment (iv) increased catalase and GSH levels but decreased TBARS and nitrite levels in brains of mice exposed to CUMS and treated with DMF .

The forced swim test is based on the fact that animals develop immobile postures when placed in an inescapable cylinder of water, and this immobility simulates clinical depression. Clinically useful antidepressants have been found to reduce periods of immobility and increase escape-directed behaviors such as vigorous activity and climbing (10, 12). The tail suspension test also produces immobility in rodents subjected to this form of stress and antidepressants decrease the immobility displayed by mice after unsuccessful attempts to escape (11, 31). In this study, treatment with DMF decreased periods of immobility in both models of depression indicative of antidepressant activity. No changes were observed in levels of both antioxidant and prooxidant biomarkers in this study following acute drug administration; we speculate that the effect of DMF in ameliorating depression in acute murine models might be independent of its' antioxidant activity. Further studies will be required to refute or validate this hypothesis.

The antidepressant activity of DMF was also evaluated in the chronic murine model of depression using the chronic unpredictable mild stress protocol as clinical management of depression requires chronic therapy. The CUMS being one of the most widely used tools for studying depression has been demonstrated to have a high predictive and construct validity prerequisites for valid animal models of psychiatric disorders (32). Exposure to uncontrollable and unpredictable stressors, which are insufficient to produce mortality, produce a state of depression in rodents similar to stress-induced clinical depression in humans $(33,34)$. The FST which is useful for evaluation of antidepressant activity following acute drug treatment is also widely used in neurobehavioral evaluation of chronic stressinduced depression in rodents (35). In this study, mice subjected to CUMS showed longer periods of immobility in the FST characteristic of behavioral despair and depression. This is similar to previous studies where stress-induced anhedonia was consistent with decrease in latency and duration of immobility in the FST $(5,36-39)$. Similar to what was observed in the acute study, low and high doses of DMF decreased periods of immobility in the forced swim test.

The sucrose preference test is also useful for evaluating chronic stress-induced neurobehavioral
A

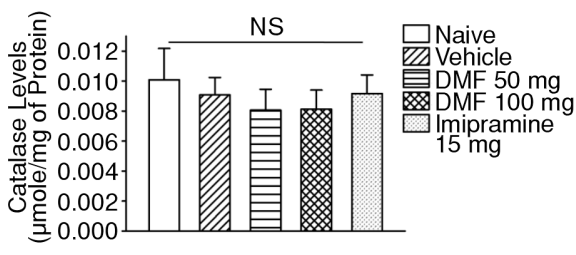

C

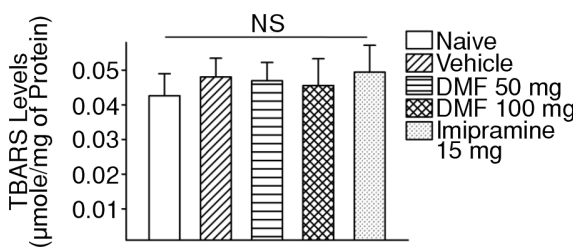

B

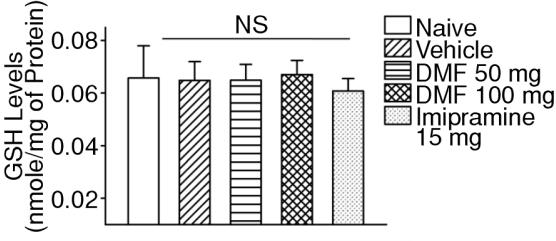

D

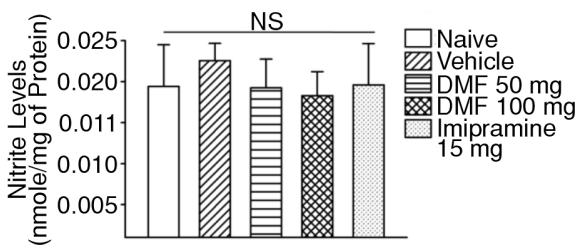

Figure 2. Statistical analysis of levels of catalase (A), Glutathione (B), thiobarbituric reactive substance (C) and nitrite levels (D) in mice following acute treatment with DMF. Acute drug treatment with DMF did not alter brain levels of antioxidant enzymes. Data is expressed as mean \pm SEM. NS means not significant; $n=6$ per group 
A

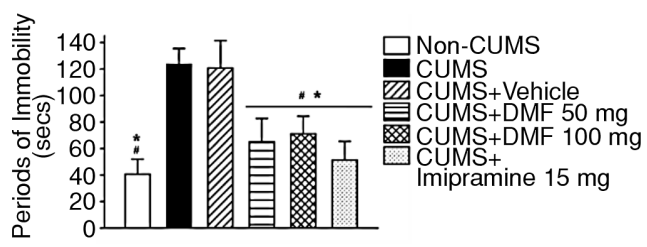

$B$

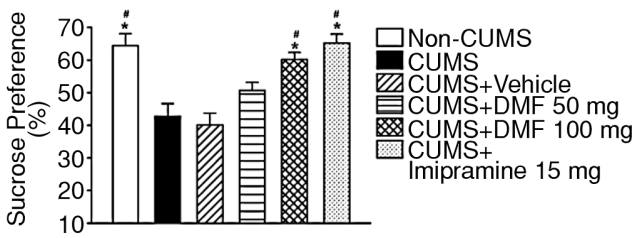

Figure 3. Statistical analysis of periods of immobility in the forced swim test (A), percentage sucrose preference (B) following exposure to chronic unpredictable mild stress and drug treatment. Administration of DMF reduced periods of immobility in the forced swim. High dose $(100 \mathrm{mg} / \mathrm{kg})$ of DMF increased sucrose consumption. Data is expressed as mean $\pm \mathrm{SEM}$. * $\mathrm{p}<0.05$ compared to the vehicle, \# $\mathrm{p}<$ 0.05 compared to CUMS group, compared to DMF $100 \mathrm{mg} / \mathrm{kg} ; \mathrm{n}=8$ per group
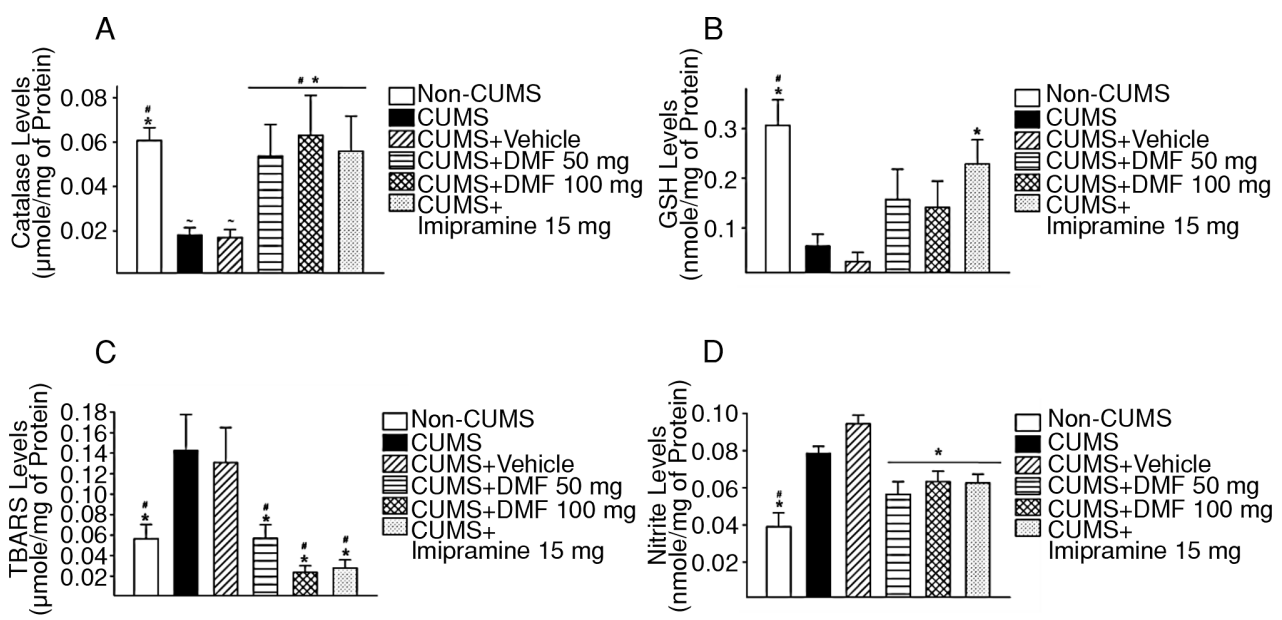

Figure 4. Statistical analysis of levels of catalase (A), Glutathione (B), TBARS (C) and nitrite levels (D) in mice following exposure to chronic mild unpredictable stress and drug treatment. DMF increased brain catalase and GSH levels but reduced levels of TBARS and nitrite in test subjects. Data is expressed as mean \pm SEM. ${ }^{*} \mathrm{p}<0.05$ compared to the vehicle, $\# \mathrm{p}<0.05$ compared to CUMS group; $\mathrm{n}=8$ per group

deficits in rodents and it is based on rodents inherent interest in sweet foods or solutions. Reduced preference of sweet solutions in the SPT represents anhedonia, one of the major symptoms of depression, and chronic but not acute treatment with antidepressants reverses this behavior $(24,25,32)$. Loss of pleasure, one of the cardinal signs of depression, is thought to be due to damage to dopaminergic and serotonergic neurons involved in the brain reward system. In a comprehensive review, Pizzagalli provided evidence showing that anhedonic stress behavior could be due to dysfunctional mesolimbic dopaminergic pathways involved in incentive motivation and reinforcement learning (40). Exposure to the CUMS protocol reduced the percentage of sucrose preference which was attenuated by high dose DMF (100 mg/kg) and imipramine. Katz, (13) and Willner et al. (24) demonstrated the ability of antidepressants to reverse CUMS induced anhedonia and consequent reduction in sucrose intake. Whether DMF interacts with dopaminergic receptors and/or dopaminergic pathways in the CNS is a hypothesis that would require further investigation.

The presence of reactive oxygen species and oxidative stress leading oxidative brain damage is implicated in the pathophysiology of depression; indeed elevated oxidative stress biomarkers were found in brains of depressed patients (16). To investigate the role of DMF in the antioxidant defense mechanism of CUMS induced depression, catalase, GSH, TBARS and nitrite levels were evaluated in brains of mice. Exposure of mice to 4 weeks of CUMS brought about an increase pro-oxidative biomarkers (TBARS and nitrite levels) and a reduction 
in brain antioxidant biomarkers (catalase and GSH) which correlated with increased periods of immobility in the FST and reduction in sucrose intake. Chronic treatment with both doses of DMF reversed the CUMS induced increase of TBARS and nitrite levels while augmenting catalase and GSH levels in the brain. Results obtained correlate with those of Grases et al., (41) and Foyet et al., (42) who demonstrated beneficial effects of reduction in oxidative stress in both clinical and preclinical settings of depression and neuropsychiatric disorders.

Despite the findings obtained, this study has some limitations which the authors hope to address in future research experiments:

(i) due to technical limitations, it was not possible to isolate the hippocampus, hence the entire brains were used for the biochemical assays

(ii) other biochemical assays for specific protein biomarkers such as Western blotting and immunohistochemistry which would have further validated some of the results obtained in this study and provided possible mechanisms of action for the effect of DMF in ameliorating depression were also not undertaken.

In summary, DMF attenuated behavioral despair in both acute and chronic models of depression and modified levels of oxidant enzymes in favor of protective antioxidants enzymes in the brains of mice subjected to chronic stress protocols and may be a potential candidate for antidepressant therapy.

\section{Authors' contribution}

The study was conceived and designed by LOI; experiments and data acquisition were carried out by LOI, EEO, OOE, EOE, and OIN. Data analysis and manuscript preparation were done by LOI and EEO. All the authors approve of the manuscript submission and accept responsibility for the entire content of this manuscript.

\section{Acknowledgments}

The authors are very grateful to Prof. Ray I. Ozolua for providing a conducive working environment for the neurobehavioral assays.

\section{Conflict of interests}

The authors declare no conflict of interest.

\section{Funding source}

The authors received no funding for this study.

\section{REFERENCES}

1. Murray C.J.L., Lopez A.D., Eds.: The Global Burden of Disease, World Health Organization, The Global Burden of Disease and Injury Series, p. 38, Genewa 2001 Available at: http://apps. who.int/iris/ bitstream/10665/ 41864/1/0965546608_eng.pdf.

2. Moussavi S., Chatterji S., Verdes E., Tandon A., Patel V., Ustun B.: Lancet 370, 851 (2007).

3. Chapman D.P., Perry G.S., Strine T.W.: Prev. Chronic Dis. 2, 1 (2005)

4. Kennedy S.H.: Eur. Neuropsychopharmacol. 16, S619 (2006).

5. Gupta D., Radhakrishnan M., Kurhe Y., Thangaraj D., Prabhakar V., Kanade P.: Acta Pharmacol. Sin. 35, 1493 (2014).

6. Maes M., Galecki P., Chang Y.S., Berk M.: Prog. Neuropsychopharmacol. Biol. Psychiatr. 29, 676 (2011).

7. Moretti M., Colla A., de Oliveira Balen G., dos Santos D.B., Budni J. et al.: J. Psychiatr. Res. 46, 331 (2012).

8. Harro J.: Preclinica 2, 402 (2004).

9. Hollis F., Kabbaj M.: ILAR J. 55, 221 (2014).

10. Cryan J.F., Markou A., Lucki I.: Pharmacol. Sci. 23, 238 (2002).

11. Steru L., Chermat R., Thierry B., Simon P.: Psychopharmacol. 85, 367 (1985).

12. Porsolt R.D.: Rev.Neurosci. 11, 53 (2000).

13. Katz R.J.: Pharmacol. Biochem. Behav. 16, 965 (1982).

14. Willner P.: Neurobiol. Stress. 6, 68 (2017).

15. Lucca G., Comim C.M., Valvassori S.S., Réus G.Z., Vuolo F. et al. J. Psychiatr. Res. 43, 864 (2009)

16. Maes M., Yirmyia R., Noraberg J., Brene S., Hibbeln J. et al.: Metab. Brain Dis. 24, 27 (2009).

17. Ellrichmann G., Petrasch-Parwez E., Lee D-H., Reick C., Arning L. et al.: PLoS ONE e161726, 1 (2011).

18. Albrecht P., Bouchachia I., Goebels N., Henke N., Hofstetter H.H. et al. J. Neuroinflammation. 9, 1 (2012).

19. Iniaghe L.O., Krafft P.R., Klebe D.W., Omogbai E.K.I., Zhang J.H., Tang J.: Neurobiol. Dis. 82, 349 (2015).

20. Kume T., Suenaga A., Izumi Y., Akaike A.: Biol. Pharm. Bull. 36, 1055 (2016).

21. Zhao X., Sun G., Zhang J., Ting S., Gonzales N., Aronowski J.: Stroke 46, 1923 (2015).

22. Public Health Service Policy on Humane Care and Use of Laboratory Animals, National Insti- 
tute of Health, USA. NIH. Available from https:/grants.nih.gov/grants/olaw/ references/ phospolicylabanimals.pdf (2015).

23. Porsolt R.D., Bertin A., Jalfre M.: Arch. Int. Pharmacodyn. 229, 327 (1977).

24. Willner P., Towell A., Sampson D., Sophokleous S., Muscat R.: Psychopharmacol. (Berl) 93, 358 (1987).

25. Serchov T., van Calker D., Biber K.: BioProtoc. 6 (2016).

26. Lowry O.H., Rosebrough N.J., Farr A.L., Randall R.J.: J. Biol. Chem. 193, 265 (1951).

27. Claiborne A.: Catalase activity in Handbook of methods for oxygen radical research. Boca Raton, Florida: CRC press. pp. 283-284 (1985).

28. Ellman G.L.: Arch. Biochem. Biophys. 82, 70 (1959).

29. Wills E.D.: Biochem. J. 99, 667 (1966).

30. Green L.C., Wagner D.A., Glogowski J., Skipper P.L., Wishnok J.S., Tannenbaum S.R.: Anal. Biochem. 126, 131 (1982).

31. O’Leary O.F., Cryan J.F. Eds., pp. 119-137 (2009). The tail-suspension test: a model for characterizing antidepressant activity in Mice. Springer protocols.

32. Willner P.: Psychopharmacol. (Berl) 83, 1 (1984).
33. Kendler K.S., Karkowski L.M., Prescott C.A.: Am. J. Psychiatr. 156, 837 (1999).

34. Anisman H., Matheson K.: Neurosci. Biobehav. Rev. 29, 525 (2005).

35. Detke M.J., Johnson J., Lucki I.: Exp. Clin. Psychopharmacol. 5, 107 (1997).

36. Strekalova T., Spanagel R., Bartsch D., Henn F.A., Gass P.: Neuropsychopharmacol. 29, 2007 (2004).

37. Kurhe Y., Radhakrishnan M., Gupta D., Devadoss T.: J. Pharm. Pharmacol. 66, 122 (2013).

38. Kudryashov N.V., Kalinina T.S.,. Voronina T.A.: Neurosci. Behav. Physiol. 46, 601 (2016).

39. Zhang L., Luo J., Zhang M., Yao W., Ma X., Yu S.Y.: Int. J. Neuropsychopharmacol. 17, 793 (2014).

40. Pizzagalli D.A.: Annu. Rev. Clin. Psychol. 10, 393 (2014).

41. Grases G., Colom M.A., Fernandez R.A., Costa-Bauzá A., Grases F.: Oxid. Med. Cell. Longev. 2014, p. 5 (2014).

42. Foyet H.S., Tchinda D.S., Koagne Y.P., Antioch I., Zingue S. et al.: BMC Complement. Altern. Med. 17, 502 (2017).

Received: 13.01.2018 